\title{
Las citocinas en la patogénesis por citomegalovirus.
}

Revisión

José D’Artagnan Villalba-Magdaleno, Ramón A. Valdés-Espinosa.

Area de Inmunología del Laboratorio Multidisciplinario de Investigación, Escuela Militar de Graduados de Sanidad, S.D.N. México, D.F., México.

\section{RESUMEN.}

La infección por Citomegalovirus Humano (CMVH) es una importante causa de morbilidad y mortalidad en pacientes inmunocomprometidos como son los pacientes con SIDA y receptores de órganos. La infección por CMVH comúnmente resulta de la reactivación del virus que se encuentra latente en el huésped. Esta reactivación de los virus genera síntomas y signos de diversas enfermedades inflamatorias tales como encefalitis, pneumonia intersticial, retinitis, hepatitis, gastritis y colitis. Los resultados de diversos experimentos en modelos murinos sugieren que las citocinas, tales como IFN- $\gamma$, TNF- $\alpha$, IL-6, IL-8, y el óxido nítrico participan en el desarrollo de estas enfermedades. Sin embargo, la razón de cómo y por qué la infección por CMVH aumenta la producción de estas citocinas es desconocido. La relación entre los niveles séricos elevados de citocinas y la detección de antígenos y ADN virales sugiere que estos factores solubles pueden contribuir a las complicaciones inmunopatológicas, por lo que la inhi- bición de la liberación o acción de estas citocinas puede ser una estrategia alternativa para prevenir la morbilidad asociada a la infección por $\mathrm{CMVH}$ en los pacientes inmunocomprometidos.

(Rev Biomed 2000; 11:293-300)

Palabras clave: citomegalovirus humano, citocinas, patogénesis.

\section{SUMMARY.}

The role of the cytokines in the pathogenesis of cytomegalovirus-associated disease.

Human cytomegalovirus (HCMV) infection is an important cause of morbidity and mortality in immunocompromised patient such as AIDS patients and organ transplant recipients. HCMV disease commonly results from the reactivation of a latent infection, causes various inflammatory diseases such as encephalitis, interstitial pneumonitis, retinitis, hepatitis, gastritis and colitis. The results of experiments from the mouse model and

Solicitud de sobretiros: M. en C. José D’Artagnan Villalba-Magdaleno. Area de Inmunología, Escuela Militar de Graduados de Sanidad, Cerrada de Palomas S/N, Col. Lomas de San Isidro, C.P. 11620, México, D.F., México. Tel. 5-540-7726 ext. 158 Fax: 5-540-0759 E-mail: jdvillalba@yahoo.com Recibido el 7/Sep./1999. Aceptado para publicación el 13/Febrero/2000. 


\section{JD'A Villalba-Magdaleno, RA Valdés-Espinosa.}

infected human suggested that the cytokines, such as IFN- $\gamma$, TNF- $\alpha$, IL-6, IL-8, and cytokineinduced nitric oxide mediate the pathogenesis of cytomegalovirus-associated disease. However, the how and why the HCMV infection enhancers the production of the cytokines is still unknown. The relation between increased cytokine plasma levels and the appearance of HCMV antigens suggests that cytokines may play an important role in the immunopathological complications. The inhibition of the release or action of those cytokines may be an alternative strategy for preventing HCMVassociated morbidity in immunocompromized patients. (Rev Biomed 2000; 11:293-300)

Key words: Human Cytomegalovirus, cytokines, pathogenesis.

\section{INTRODUCCIÓN.}

El Citomegalovirus humano (CMVH) es un betaherpesvirus de distribución mundial, responsable de una importante morbilidad y mortalidad en recién nacidos infectados in utero y pacientes inmunocomprometidos. Aproximadamente el $95 \%$ de los individuos adultos en los países en vías de desarrollo y del 60 al 80\% de los que habitan en los países desarrollados están infectados por CMVH (1). Después de una infección primaria el CMVH establece una infección persistente que dura el resto de la vida del huésped. Aunque la inmunidad celular puede proteger de la reactivación del CMVH y de las enfermedades asociadas a él, éste raramente es eliminado del huésped. La infección por CMVH en individuos inmunocomprometidos, como los neonatos, los pacientes con cáncer, los receptores de un trasplante de órganos tratados con inmunosupresores y enfermos con SIDA (Síndrome de Inmunodeficiencia Adquirida), causa varias enfermedades inflamatorias tales como encefalitis, pneumonitis intersticial, retinitis, hepatitis, gastritis, colitis y alteraciones hematológicas (2). La mayoría de las enfermedades asociadas a CMVH son el resultado de la reactivación de vi- rus latente o persistente adquiridos antes de la inmunosupresión.

Las citocinas son de importancia central en la regulación de diferentes eventos celulares, como son los inmunológicos, los inflamatorios, la regeneración de tejidos y el desarrollo embrionario. Ellas comprenden un grupo de proteínas de bajo peso molecular que son producidas por diferentes tipos celulares y generalmente actúan de una manera parácrina o autócrina, su producción es transitoria y compleja, y se encuentra sujeta a un estricto control (3). Las citocinas raramente son liberadas individualmente. Una citocina es capaz de estimular la producción de muchas otras generando una red que interacciona con otros reguladores celulares como son las hormonas y neuropéptidos. El mejor entendimiento de la red de citocinas y su manipulación en las enfermedades tiene un considerable potencial clínico. Para entender el papel de la respuesta inmune del huésped en el control de la infección por CMVH y el desarrollo de diferentes síndromes asociados, es conveniente describir cómo se inicia una respuesta inmune ante una infección por CMVH y cómo participan las citocinas en el desarrollo de otras enfermedades que acompañan a la infección por este virus.

\section{Respuesta inmune contra CMVH.}

La defensa inicial contra la invasión viral comienza con la respuesta inmune innata o no específica que incluye a diferentes citocinas con propiedades de regular la respuesta inmune (celular y humoral) antiviral, también las citocinas pueden tener efectos antivirales directos, tal es el caso del factor de necrosis tumoral- $\alpha$ (TNF- $\alpha$ ) e interferón- $\gamma($ IFN- $\gamma)$ que actúan sobre las células infectadas impidiendo la replicación viral (Fig. 1). Se conoce que las citocinas tipo I, tienen una participación muy importante como moléculas efectoras antivirales en la respuesta por células $T$ contra las infecciones virales (4).

En infecciones con citomegalovirus murino (CMVM) se inducen altos niveles de interleucina12 (IL-12), IFN- $\gamma$, TNF- $\alpha$ e IL-6, citocinas que

\section{Revista Biomédica}


Citocinas y citomegalovirus.

están asociadas con efectos pro-inflamatorios $(5$, 6). Las citocinas son de mucha importancia para el control de la infección por CMVH y otros virus, como se ha demostrado en numerosos experimentos, por ejemplo, la expresión de citocinas tipo I en vectores, como el virus recombinante de la vaccinia (rVV), crea un virus con menor patogenicidad en ratones normales e inmunodeficientes, estas incluyen IL-2, IL-12, IFN- $\gamma$ y TNF- $\alpha$ (7). La menor patogenicidad de estos virus es debida a la capacidad de estos factores solubles de suprimir el crecimiento viral in vivo más que aumentar la respuesta antiviral del huésped. La importancia de los interferones y los factores de necrosis tumorales durante la infección viral también ha sido demostrada usando ratones deficientes en la expresión de estas citocinas o de sus receptores. Los ratones deficientes en la expresión de IFN- $\gamma$, el receptor de IFN- $\gamma$ (IFN- $\gamma$ R), el IFN- $\alpha / \beta R$, los receptores de TNF (TNFRs) o IL-6 son extremadamente susceptibles a la infección viral.
La expresión de IL-12 en el rVV resulta un virus atenuado, sin embargo, el efecto protector de este factor es completamente revertido en ratones deficientes del IFN- $\gamma \mathrm{R}$, demostrando con esto que la actividad antiviral observada con esta citocina esta mediada por IFN- $\gamma$; y esto se debe a que IL12 promueve fuertemente la diferenciación de células T CD4+ al fenotipo Th1 y suprime la expresión de citocinas Th2, además aumenta las funciones líticas de linfocitos T citotóxicos (LTCs) y de células Natural Killer (NKs) y en forma importante incrementa la capacidad de estas células para la liberación de IFN- $\gamma(8)$.

\section{Inmunopatologías asociadas a una infección por CMVH.}

El CMVH es un patógeno importante en individuos inmunocomprometidos, aquellos con SIDA, receptores de trasplante de médula ósea o de un órgano sólido. También los pacientes con cáncer, sepsis y neonatos, son blancos importantes

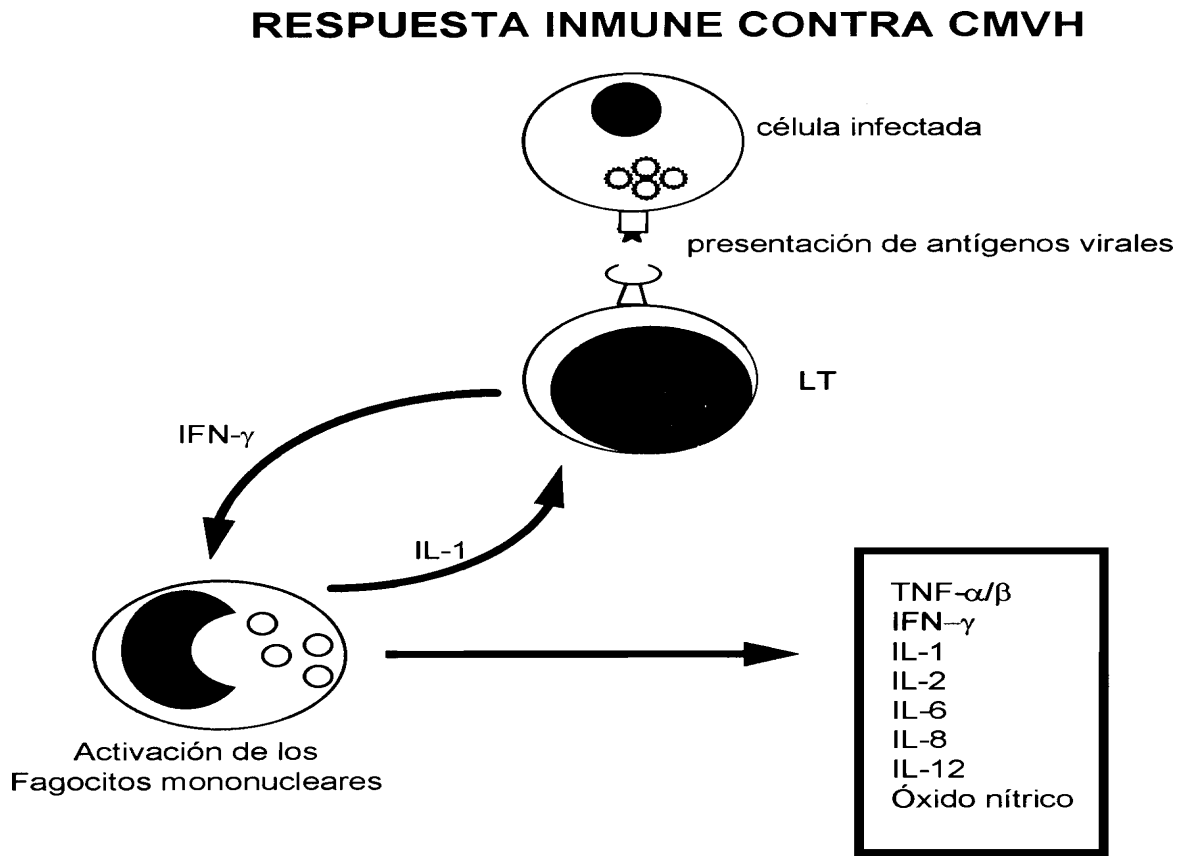

Figura 1.- Las proteínas virales son procesadas en el citoplasma de la célula infectada generando péptidos que son presentados por el Complejo Principal de Histocompatibilidad clase I a los linfocitos T (LT), los cuales liberan IFN- $\gamma$ al microambiente para activar a los macrófagos quienes secretan citocinas del tipo I y óxido nítrico. 


\section{JD'A Villalba-Magdaleno, RA Valdés-Espinosa.}

para iniciar la reactivación de CMVH y con ello desarrollar enfermedades inflamatorias asociadas a esta infección. Diferentes investigaciones sobre la patogénesis de infecciones por CMVH revelaron una asociación de síntomas y signos en varios grupos de pacientes. Las investigaciones para la prevención o tratamiento de las enfermedades asociadas a una infección por este virus son de mucha importancia para entender si CMVH es un agente causal o concomitante. Los estudios histopatológicos, inmunohistoquímicos o por hibridación in situ han ayudado a definir el grado de replicación viral y expresión de citocinas en varios tipos de células y órganos de individuos infectados, habiéndose encontrado una irregularidad en la secreción de citocinas con una mínima o ausente citotoxicidad mediada por células $\mathrm{T}(9,10)$. A continuación se describen varios resultados que sugieren la participación de diferentes factores solubles como responsables en la generación de nuevas complicaciones clínicas en los individuos que presentan infección por CMVH.

El CMVH causa varias enfermedades inflamatorias que se presentan principalmente en pacientes inmunocomprometidos. El desarrollo de estas enfermedades no se debe de manera directa a la replicación viral, sino a las citocinas producidas por el sistema inmune (Cuadro 1). En el modelo murino, se encontró que cuatro semanas después de las inoculaciones intraperitoneales de CMVM, el virus sólo fue detectable en las glándulas salivales, pero no en los pulmones y otros órganos. Cuando las células $\mathrm{T}$ de estos ratones fueron activadas in vivo con un anticuerpo monoclonal anti-CD3, se generó un cuadro de pneumonitis intersticial (PI) con una excesiva producción de citocinas. En los pulmones de los ratones infectados, los ARN mensajeros (ARNm) de citocinas como IL-2, IL-6, IFN- $\gamma$ y TNF- $\alpha$ fueron abundantemente expresados tres horas después de la inyección de anti-CD3. Además, se encontró expresión de la sintetasa inducible del óxido nítrico y niveles incrementados de óxido nítrico $(\mathrm{ON})$ en los pulmones; la administración de antagonistas del ON alivió la pneumonitis, concluyendo que la patología pulmonar asociada a CMVM es mediada por diversas citocinas y por el ON inducido por éstas (11). Posteriormente Tanaka, sugirió de igual forma, que la PI asociada a CMVM era desarrollada por citocinas, como el IFN- $\gamma$ y TNF- $\alpha$ y el ON inducido por estos factores solubles (12). En los pacientes con trasplante de pulmón, quienes desarrollan pneumonía por citomegalovirus, esta se acompaña por una intensa actividad inflamatoria local y sistémica, encontrándose en el suero de estos pacientes mediadores solubles que pueden participar en el desarrollo de una respuesta inflamatoria aguda o crónica la cual puede ser potencialmente agresiva para el tejido trasplantado. Entre las citocinas y los receptores de éstas que se han identificado, están el receptor soluble de IL-2 (IL2sR), IFN- $\gamma$, TNF-sR55 y TNF-sR75, IL-6 e IL$1 \beta$ (13). La producción de RANTES (regulated

Cuadro 1

Papel de las citocinas en la patogénesis de enfermedades asociadas a Citomegalovirus

\begin{tabular}{lll}
\hline Patología & Citocina & Referencia \\
\hline Pneumonia & TNF- $\alpha$ & 12 \\
Intersticial & TNF-sR55 & 11 \\
& TNF-sR75 & \\
& IFN- $\gamma$ & 12,11 \\
& IL-1 $\beta$ & 11 \\
& IL-2 & 11 \\
& IL-2sR & \\
& IL-6 & 14 \\
& RANTES & 21 \\
Artritis Reumatoide & IL-6 & \\
& IL-8 & 22 \\
Retinitis & TNF- $\alpha$ & 19 \\
Hepatitis & TNF- $\alpha$ & 24 \\
Colitis & IL-2 & 20 \\
Pancreatitis & IL-1 & \\
\hline & TNF- $\alpha$ & \\
& &
\end{tabular}

\section{Revista Biomédica}


Citocinas y citomegalovirus.

upon activation, normally $T$ expressed and secreted) en los fluidos broncoalveolares es significativamente más alta durante la PI por CMVH (36.2 $+/-16 \mathrm{pg} / \mathrm{mL}$ ) que en los pacientes sin complicaciones $(9.1+/-2.3 \mathrm{pg} / \mathrm{mL})$. Los macrófagos pulmonares de pacientes con pneumonitis cultivados por 24 horas liberan espontáneamente grandes cantidades de RANTES $(140+/-53$ pg/mL) comparados con los macrófagos de pacientes control $(15.2+/-6.5 \mathrm{pg} / \mathrm{mL})$, por lo que la producción intrapulmonar de esta quimiocina por los macrófagos activados contribuye a la acumulación de células productoras de citocinas, ocasionando complicaciones como se ha observado en los trasplantes de diferentes órganos (14).

El CMVH es una causa importante de morbilidad y mortalidad en los receptores de un trasplante de órgano. La infección por este virus frecuentemente resulta de la reactivación de una infección latente. El rechazo del injerto e infección por CMVH son las dos complicaciones principales en los alotrasplantes. La infección por CMVH puede promover el rechazo por diferentes mecanismos que incluyen la producción de citocinas inflamatorias, incrementando la expresión de moléculas de histocompatibilidad y de adhesión (15). Fietze y colaboradores reportaron la detección de antígenos y ADN de CMVH en células de sangre periférica provenientes de receptores de diferentes órganos, y altos niveles séricos de TNF- $\alpha$, sugiriendo que esta citocina puede participar en el balance entre la latencia y la reactivación de CMVH, y que la inhibición de su liberación o acción puede ser una estrategia para prevenir la morbilidad asociada a la infección por CMVH en estos pacientes (16). Esta hipótesis es apoyada por el hallazgo de que TNF- $\alpha$ es capaz de estimular la actividad de factores de transcripción que actúan en las regiones promotoras de los genes IE (inmediatos tempranos) de este virus. Recientemente Humar y colaboradores reportaron elevadas concentraciones séricas de algunas citocinas en pacientes con trasplante de médula ósea que presentaban infección por CMVH . Los niveles de IL-6 (281.2
+/-85.5 vs. $95.7+/-15.0 \mathrm{pg} / \mathrm{mL}$ ) fueron significativamente más altos en estos pacientes que en aquellos que no desarrollaron la enfermedad. Los niveles de IL- 8 y TNF- $\alpha$ fueron también elevados en los pacientes con la enfermedad activa (17). Al igual que TNF- $\alpha$, Mukaida reportó que IL- 8 también puede activar a NF- $\mathrm{BB}$ y con ello la replicación de citomegalovirus y la producción de viriones infectivos (18).

Orange por su parte, demostró la participación de TNF- $\alpha$ en la patología hepática durante la infección en el ratón por CMVM, tanto en animales inmunocompetentes, como en los ratones E26 deficientes de células T y NKs, el virus indujo una enfermedad hepática caracterizada por inflamación local y expresión de antígenos virales. Mediante el uso de anticuerpos neutralizantes dirigidos contra esta citocina se demostró que además de los efectos antivirales, participó en el desarrollo de focos necróticos. En los ratones inmunodeficientes E26, la enfermedad hepática inducida por CMVM es progresiva, con eventuales muertes. El bloqueo de TNF- $\alpha$ significativamente incrementó la sobrevivencia de estos animales, por lo tanto, TNF- $\alpha$ participa en las lesiones hepáticas independiente de las funciones efectoras de células T y NKs (19).

Las infecciones por CMVM están implicadas en la diabetes mellitus dependiente de insulina, los efectos de la infección por CMVM sobre el páncreas reflejan una inflamación local de este órgano, y el tratamiento in vivo con receptores solubles de citocinas sugirieron que IL-1 y/o TNF$\alpha$ contribuyen a la necrosis acinar (20). Otros reportes mostraron una correlación entre los niveles elevados de citocinas y la presencia del genoma de CMVH en un proceso inflamatorio como lo es la artritis reumatoide. Murayama y colaboradores encontraron en el líquido sinovial de pacientes con artritis reumatoide, ADN de CMVH y elevados niveles de IL-8 e IL-6 con valores diez veces mayores con respecto a los pacientes control (21). La retinitis causada por CMVH es otra de las enfermedades que se le asocian a este virus, el cual daña a las células endoteliales oculares causando 
JD'A Villalba-Magdaleno, RA Valdés-Espinosa.

una vasculitis oclusiva y generando problemas reológicos sanguíneos. En los pacientes infectados con VIH la infección con CMVH se ha asociado con elevados niveles de TNF- $\alpha$ (22). La inmunoterapia con citocinas podría reducir la frecuencia de retinitis causada por $\mathrm{CMVH}$, en su trabajo Dix y colaboradores demostraron que la infección ocular con CMVM fue reducida en los ratones inmunodeficientes tratados con IL-2, pero no en los ojos de aquellos animales tratados con IL-12. Estos resultados apoyaron la hipótesis de que la inmunoterapia con concentraciones de citocinas controladas reduce la retinitis por CMVM en estos ratones inmunosuprimidos (23). Por su parte, Presti demostró que IFN- $\gamma$ es necesario para regular no solo la infección viral, sino la arteritis asociada a CMVM. Los ratones carentes del receptor para esta citocina desarrollaron inflamación aórtica crónica, con cuerpos de inclusión y antígenos virales (24). Entre los síndromes debidos a CMVH en el huésped inmunodeprimido se encuentra el daño al aparato digestivo que puede ser localizado o extenso. También pueden presentarse úlceras en esófago, estómago, intestino delgado o colon los cuales pueden ocasionar hemorragia o perforación. La infección por CMVH puede desencadenar la exacerbación de una colitis ulcerosa subyacente (2).

\section{CONCLUSIONES.}

El CMVH infecta a la mayoría de la población de adultos, la infección primaria es frecuentemente acompañada por una neutropenia y trombocitopenia y es seguida por un período asintomático (25). En los pacientes inmunocomprometidos como los que reciben un trasplante de médula ósea y otros órganos o tejidos, los individuos con cáncer y SIDA así como los recién nacidos, existen condiciones que favorecen la infección o reactivación viral causando serios problemas de salud. El desarrollo de diferentes síndromes que se asocian a la infección por CMVH están influenciados por el sistema inmunológico del huésped. Las enfermedades asociadas a la infección por CMVH van desde una hepatitis o daño gastrointestinal hasta una pneumonitis o anormalidades hematológicas. Diversas citocinas participan en la infección y replicación por $\mathrm{CMVH}$; por ejemplo, IL-1, TNF- $\alpha$, IFN- $\gamma$ e IL-12 son algunas citocinas que están implicadas en reducir y controlar la replicación viral, sin embargo, la funcionalidad de estas citocinas continúan aún después de cumplir sus funciones en la activación de células T CD8 ${ }^{+}$ que participan en la eliminación del virus, y de esta manera las citocinas puedan generar la patogénesis indirectamente. Probablemente la patogénesis de estos síndromes sea multifactorial y por lo tanto se requiere de nuevas estrategias alternativas para prevenir la morbilidad asociada a CMVH. El TNF- $\alpha$ e IFN- $\gamma$ están bien documentados por tener una actividad inmunomoduladora y antiviral en el control de la infección por CMVH, Pomeroy explica que el tratamiento con IFN- $\gamma$ exógeno en ratones infectados con CMVM dos días después de iniciar la infección, tuvo un efecto protector a dosis bajas, mientras que la terapia con IFN- $\gamma$ incrementó la morbilidad y mortalidad cuando se emplearon altas dosis (26), por lo tanto, IFN- $\gamma$ tiene un papel muy importante en la respuesta inmunológica en contra de CMVM y presenta efectos benéficos y adversos dependientes de la dosis y tiempo.

Como mediadores solubles en los procesos inmunológicos, inflamatorios y de crecimiento celular, las citocinas empiezan a ser usadas en casos de enfermedades malignas, infecciones virales crónicas, infecciones parasitarias y en algunas enfermedades inmunes congénitas. Paradójicamente, la producción descontrolada o excesiva puede contribuir a la patofisiología de algunas infecciones agudas o crónicas, enfermedades autoinmunes y neoplasias. De esta manera, antagonistas de citocinas, como anticuerpos o péptidos que las mimeticen, pueden tener un potencial terapéutico en las enfermedades inflamatorias que pueden presentarse en los individuos inmunocomprometidos, asociados a la infección por CMVH.

\section{Revista Biomédica}




\section{Citocinas y citomegalovirus.}

\section{REFERENCIAS.}

1.- Griffiths PD, Grundy JE. Molecular biology and immunology of cytomegalovirus. Biochem J 1987; 241:313.

2.- Hirsch SM. Infección por citomegalovirus. En Isselbacher KJ, Braunwald E, Wilson JD, Martin JB, Fauci AS, Kasper DL. Principios de medicina interna de Harrison. 13a. de. Interamericana Mc-Graw-Hill de España; 1994; 146:922-6.

3.- Arai K. Cytokines: coordinators of immune and inflammatory responses. Annu Rev Biochem 1990; 59:783836.

4.- Ramsay AJ, Ruby J, Ramshaw IA. A case for cytokines as effector molecules in the resolution of virus infection. Immunol Today 1993; 14:155-7.

5.- Yerkovich ST, Olver SD, Lenzo JC, Peacock CD, Price $\mathrm{P}$. The roles of tumour necrosis factor-, interleukin-1 and interleukin-12 in murine cytomegalovirus infection. Immunology 1997; 91:45-52.

6.- Biron ChA, Cousens LP, Ruzek MC, Su HC, SalazarMather TP. Early cytokine responses to viral infections and their roles in shaping endogenous cellular immunity. En Mechanisms of lymphocyte activation and immune regulation VII Plenum Press, New York; 1998; 15:143-9.

7.- Ramshaw IA, Ramsay AJ, Karupiah G, Rolph MS, Mahalingam S, Ruby JC. Citokines and immunity to viral infection. Immunol Rev 1997; 159:119-35.

8.- Trinchieri G. Interleukin-12: a proinflammatory cytokine with immunoregulatory function that bridge innate resistance and antigen-specific adaptive immunity. Annu Rev Immunol 1995; 13:251-76.

9.- Podlech J, Holtappels R, Wirtz N, Steffens HP, Reddehase MJ. Reconstitution of CD8+ T cells is essential for the prevention of multiple-organ cytomegalovirus histopathology after bone marrow transplantation. J Gen Virol 1998; 79(Pt 9):2099-104.

10.- Kern F, Ode-Hakim S, Nugel H, Vogt K, Volk HD, Reinke P. Peripheral $\mathrm{T}$ cell activation in long-term renal transplant patient: concordant upregulation of adhesion molecules and cytokine gene transcription. J Am Soc Nephrol 1996; 7:2476-82.

11.- Tanaka K, Nakazawa H, Okada K, Umezawa K, Fukuyama N, Koga Y. Nitric oxide mediates murine cytomegalovirus-associated pneumonitis in lung that are free of the virus. J Clin Invest 1997; 100: 1822-30.

12.- Tanaka K. The role of the host's immune system in the pathogenesis of cytomegalovirus-associated disease. Nippon Rinsho 1998; 56:97-101.

13.- Humbert M, Emilie D. Immunopathology of cytomegalovirus pneumonia and allograft rejection in lung transplantation. Rev Mal Respir 1994; 11:559-66.

14.- Monti G, Magnan A, Fattal M, Rain B, Humbert M, Mege JL, Noirclerc M, Dartevelle P, Cerrina J, Simonneau G, Galanaud P, Emilie D. Intrapulmonary production of RANTES during rejection and CMV pneumonitis after lung transplantation. Transplantation 1996; 61:1757-62.

15.- Humbert M, Emilie D. Cytomegalovirus infection and allograft rejection. Rev Mal Respir 1996; 13:5 Suppl S8591.

16.- Fietze E, Prösch S, Reinke P, Stein J, Döcker WD, Staffa G, Löning S, devaux S, Emmrich F, von Baehr R. Cytomegalovirus infection in transplant recipients. The role of tumor necrosis factor. Transplantation 1994; 58:675-80.

17.- Humar A, St Louis P, Mazzulli T, McGeer A, Lipton J, Messner H, MacDonald KS. Elevated serum cytokines are associated with cytomegalovirus infection and disease in bone marrow transplant recipients. J Infect Dis 1999; 179:484-8.

18.- Mukaida N, Murayama T. Molecular mechanism of interleukin-8 gene expression. Rinsho Byori 1998; 46:8218.

19.- Orange JS, Salazar-Mather TP, Opal SM, Biron CA. Mechanisms for virus-induced liver disease: tumor necrosis factor-mediated pathology independent of natural killer and $\mathrm{T}$ cells during murine cytomegalovirus infection. J Virol 1997; 71:9248-58.

20.- Price P, Baxter AG, Allcock RN, Papadimitriou JM. Factors influencing the effectos of murine cytomegalovirus on the pancreas. Eur J Clin Invest 1998; 28:546-53.

21.- Murayama T, Tsuchiya N, Jisaki F, Ozaki M, Sakamuro D, Hirai K, Shimizu S, Ito K, Matsushima K, Furukawa T. Elevated cytokine levels in synovial fluid of rheumatoid arthritis correlates with the presense of cytomegalovirus genome. Autoimmunity 1994; 17:333-7. 
$J D^{\prime A}$ Villalba-Magdaleno, RA Valdés-Espinosa.

22.- Conway MD, Tong P, Olk RJ. Branch retinal artery occlusion (BRAO) combined with branch retinal vein occlusion (BRVO) and optic disc neovascularization associated with HIV and CMV retinitis. Int Ophthalmol 1995-96; 19:249-52.

23.- Dix RD, Giedlin M, Cousins SW. Systemic cytokine immunotherapy for experimental cytomegalovirus retinitis in mice with retrovirus-induced immunodeficiency. Invest Ophthalmol Vis Sci 1997; 38:1411-7.

24.- Presti RM, Pollock JL, Dal Canto AJ, O’Guin AK, Virgin HW. Interferon gamma regulates acute and latent murine cytomegalovirus infection and chronic disease of the great vessels. J Exp Med 1998; 188:577-88.

25.- Cinatl J Jr, Cinatl J, Vogel JU, Rabenau H. Modulatory effects of human cytomegalovirus infection on malignant properties of cancer cells. Intervirology 1996; 39:259-69.

26.- Pomeroy C, Delong D, Clabots C, Riciputi P, Filice GA. Role of interferon-gamma in murine cytomegalovirus infection. J Lab Clin Med 1998; 132:124-33. 